\title{
Étanchéités statiques des écoulements de filtration au voisinage du point critique
}

\author{
Raouf Khelalfa $^{1, a}$, Jean-Sylvestre Darrozès ${ }^{2}$ et Jean-Félix Durastanti ${ }^{1}$ \\ 1 Centre d'Études et de Recherche en Thermique, Environnement et Systèmes, Paris 12, \\ 61 avenue du Général de Gaulle, 94010 Créteil Cedex, France \\ 2 Institut Jean Le Rond d'Alembert, Paris 6, 4 place Jussieu, 75252 Paris Cedex 05, France
}

Reçu le 1 février 2010, accepté le 29 novembre 2010

\begin{abstract}
Résumé - L'assemblage de certaines pièces métalliques qu'on peut rencontrer dans l'industrie (nucléaire, spatiale, ...), engendre souvent un chemin de fuite qui peut laisser passer un fluide. L'étude suivante a pour objet la compréhension de la phénoménologie d'un écoulement confiné traversant le voisinage du point critique et son influence sur le débit de fuite. Ce problème d'étanchéité a été limité aux situations stationnaires et visqueuses. Le chemin de filtration a été assimilé à un capillaire dont la paroi est maintenue à la température critique. Une différence de pression a été imposée entre une entrée supercritique et une sortie subcritique. L'approche phénoménologique a permis de mettre en évidence la présence d'une zone de transition au passage du point critique. Dans cette région de forte dilatation on a montré l'existence d'un couplage thermo-dynamique et le rôle joué par la convection thermique qui associe la conduction pour assurer le transport de chaleur. On montre que la progression du fluide vers l'extérieur est ralentie par un effet bouchon.
\end{abstract}

Mots clés : Étanchéités statiques / point critique / débit de fuite / capillaire micrométrique / approche phénoménologique / couplage thermo-mécanique / dilatation / effet bouchon

\begin{abstract}
Static seals of confined flow near critical point. The assembly of metal parts that may be encountered in industry (nuclear, space, ...), creates frequently a path of leakage which can let a fluid pass. In this following study, we aim at understanding the phenomenology of a confined flow through the vicinity of critical point and its influence on the leakage rate. This sealing problem was limited to stationary and viscous situations. The leakage geometry has been assimilated to a capillary tube whose the wall temperature is fixed at the fluid critical value. A difference of pressure was imposed between a supercritical inlet and a subcritique outlet. The phenomenological approach has led to highlight the existence of a transition zone crossing the critical point. In this region of strong expansion, has shown the existence of a thermo-mechanical coupling and be taken a into account by the thermal convection joined the conduction to transport heat. It shows that the progression in fluid outwards is slowed by that cap effect.
\end{abstract}

Key words: Static sealing / critical point / mass flow / micrometric capillaries / phenomenological approach / thermo-mechanical coupling / expansion / cap effect

\section{Introduction}

En industrie chimique, pétrochimique ou nucléaire certains montages de pièces sont assurés par des raccords. Dans ces montages le contact imparfait entre deux surfaces rugueuses engendre un espace de filtration à géométrie complexe. Pour répondre aux interrogations des industriels sur ce problème d'étanchéité [1] et parti-

\footnotetext{
a Auteur pour correspondance :

raouf.khelalfa@univ-paris12.fr
}

culièrement dans les conditions extrêmes d'exploitation, une étude a été entreprise dans ce sens. Dans ce qui va suivre, le chemin de fuite est réduit à un tube capillaire aux extrémités duquel une différence de pression est imposée entre une entrée supercritique et une sortie subcritique. Les données ont été prises de telle sorte que le fluide soit dominé par la viscosité créant ainsi un écoulement rampant. Par ailleurs, comme ce travail s'inscrit dans le cadre des évolutions compressibles traversant le voisinage du point critique [2-4], les propriétés physiques de la phase gazeuse, de la phase liquide et de la phase 


\section{Nomenclature}

\begin{tabular}{|lll|}
\hline$c_{\mathrm{p}}$ & Chaleur spécifique & $\mathrm{J} \cdot \mathrm{kg}^{-1} \cdot \mathrm{K}^{-1}$ \\
$E_{0}$ & Nombre d'Eckert & \\
$f$ & Vecteur force de volume & \\
$k$ & Conductivité thermique & $\mathrm{W} \cdot \mathrm{m}^{-1} \cdot \mathrm{K}^{-1}$ \\
$L$ & Longueur du capillaire & $\mathrm{m}$ \\
$p$ & Pression & $\mathrm{Pa}$ \\
$P r_{0}$ & Nombre de Prandtl & $\mathrm{kg} \cdot \mathrm{s}^{-1}$ \\
$Q$ & Débit massique & $\mathrm{m}$ \\
$r$ & Coordonnée radiale & $\mathrm{m}$ \\
$R$ & Rayon du capillaire & $\mathrm{K}$ \\
$R e_{\mathrm{R}}$ & Nombre de Reynolds & $\mathrm{m} \cdot \mathrm{s}^{-1}$ \\
$T$ & Température & $\mathrm{m}$ \\
$U_{0}$ & Vitesse de Poiseuille & $\mathrm{K}$ \\
$\boldsymbol{V}$ & Vecteur de vitesse $=u \boldsymbol{e}_{r}+v \boldsymbol{e}_{z}$ \\
$z$ & Coordonnée longitudinale & \\
$\beta$ & Coefficient de dilatation & \\
$\delta_{x}$ & Échelle de la grandeur $x$ & \\
$\varepsilon$ & Facteur géométrique & \\
$\eta$ & Épaisseur de la couche limite & \\
$\lambda$ & Viscosité volumique & $\mathrm{kg} \cdot \mathrm{m}^{-1} \cdot \mathrm{s}^{-1}$ \\
$\mu$ & Viscosité dynamique & $\mathrm{kg} \cdot \mathrm{m}^{-1} \cdot \mathrm{s}^{-1}$ \\
$\rho$ & Masse volumique & $\mathrm{kg} \cdot \mathrm{m}^{-3}$ \\
\hline & & \\
\hline 0 & Quantité de référence & \\
$\mathrm{c}$ & Critique & \\
$\mathrm{E}$ & Entrée & \\
$\mathrm{p}$ & Pression constante & \\
$\mathrm{r}$ & Radiale & \\
$\mathrm{S}$ & Sortie & \\
\hline
\end{tabular}

supercritique tendent à se confondre dans cette région. On montre dans cet article que le passage par l'état critique s'effectue par l'intermédiaire d'une zone de transition [5] de forte dilatation sous le contrôle d'un couplage thermo - dynamique mis en évidence par la modélisation de cette région intérieure. Le mécanisme de transport de chaleur dans ce tronçon est assuré conjointement par la conduction thermique (observée au départ dans la région extérieure amont) et la convection qui devient importante dans ce milieu critique. Sur le plan dynamique, la position de la couche limite libre influe sur la valeur du débit de fuite. Précisément, plus cette zone dilatable se rapproche de la sortie du conduit plus la quantité du fluide qui sort à l'extérieur par unité de section diminue, d'où un effet bouchon.

\section{Position du problème}

On recherche le comportement d'un fluide dans un milieu confiné lorsque sur son chemin son état thermodynamique traverse le voisinage du point critique $\left(p_{\mathrm{c}}, \rho_{\mathrm{c}}, T_{\mathrm{c}}\right)$ où certains coefficients de transport divergent [6]. Pour comprendre les phénomènes essentiels, on écarte les complexités physiques et géométriques en se plaçant dans le cadre d'hypothèses simplificatrices.

Soit un écoulement s'effectue dans une conduite de section circulaire de sorte que le problème est axisymétrique. Son rayon $R$ dans le sens radial est petit devant sa longueur $L$ dans la direction longitudinale, de sorte que le facteur géométrique $R / L$ est très petit devant l'unité et noté $\varepsilon$. Pour cet écoulement qui traverse une zone de transition très proche du point critique où les phases liquide, fluide supercritique et gaz sont confondues, la tension superficielle est négligeable. Le fluide en régime stationnaire et compressible est dominé par la viscosité (laminaire établi). Ce mouvement est créé par une différence de pression $p_{\mathrm{E}}-p_{\mathrm{S}}$ qu'on applique entre l'entrée (E) et la sortie (S) du capillaire (Fig. 1). Respectivement en amont et en aval, la pression est supérieure $\left(p_{\mathrm{E}}>p_{\mathrm{c}}\right)$ et inférieure $\left(p_{\mathrm{S}}<p_{\mathrm{c}}\right)$ à la valeur critique. Le conduit est maintenu à la température critique $\left(T_{\mathrm{p}}=T_{\mathrm{c}}\right)$.

Dans ce problème de fuite (Fig. 1) en étanchéité statique, l'étude du comportement de l'écoulement traversant le voisinage du point critique qui est essentiellement de nature dynamique $[2,4]$ est traitée ici par une approche mécanicienne. L'évolution du fluide est donc régie dans ce cadre par les équations de Navier-Stokes compressibles en régime stationnaire obéissant à la loi thermodynamique de Van der Waals (VDW). Sous forme intrinsèque, on a :

$$
\begin{aligned}
& \boldsymbol{\nabla} . \rho \boldsymbol{V}=0 \\
& \rho(\boldsymbol{V} \cdot \boldsymbol{\nabla}) \boldsymbol{V}=-\boldsymbol{\nabla} p+\boldsymbol{\nabla}[(\lambda+\mu) \boldsymbol{\nabla} \cdot \boldsymbol{V}]+\boldsymbol{\nabla} \cdot(\mu \boldsymbol{\nabla} \boldsymbol{V})+\rho \boldsymbol{f} \\
& \rho c_{\mathrm{p}} \boldsymbol{V} \cdot \boldsymbol{\nabla} T-\boldsymbol{\nabla} \cdot(k \boldsymbol{\nabla} T)=\beta T \boldsymbol{V} \cdot \boldsymbol{\nabla} p+2 \mu(\underline{\underline{D}}: \underline{\underline{D}})+\lambda(\boldsymbol{\nabla} \cdot \boldsymbol{V})^{2} \\
& p=\frac{\rho r_{v} T}{1-\rho b}-a \rho^{2}
\end{aligned}
$$

$u$ et $v$ désignent respectivement la composante longitudinale et radiale du champ de vitesse en coordonnées cylindriques. La viscosité dynamique $\mu$ et volumique $\lambda$ du fluide dépendent en général de la température et sont reliées par l'approximation de Stokes :

$$
\lambda(T)+\frac{2}{3} \mu(T)=0
$$

Dans le modèle de (VDW) où le caractère singulier du point critique est correctement décrit, l'approximation de l'état thermodynamique réel du fluide dans cette région est jugée qualitativement acceptable dans le cadre d'une approche d'analyse de grandeurs qui est ici la nôtre. $a, b$ et $r_{v}$ sont des constantes de la loi d'état (VDW), fonction des coordonnées thermodynamiques critiques $\left(p_{\mathrm{c}}, T_{\mathrm{c}}, \rho_{\mathrm{c}}\right)$ telles que :

$$
a=\frac{3 p_{\mathrm{c}}}{\rho_{\mathrm{c}}^{2}} \quad b=\frac{1}{3 \rho_{\mathrm{c}}} \quad r_{v}=\frac{8 p_{\mathrm{c}}}{3 \rho_{\mathrm{c}} T_{\mathrm{c}}}
$$

Dans le cas de l'approximation de Van der Waals, le coefficient de dilatation isobare $\beta$ et la capacité calorifique à pression constante $c_{\mathrm{p}}$ ont respectivement pour expression $[5,6]$ :

$$
\begin{aligned}
\beta & =\frac{r_{v} \rho}{p-a(1-2 b \rho) \rho^{2}} \\
c_{\mathrm{p}} & =c_{\mathrm{p}_{0}}+\frac{2 a r_{v}(1-b \rho) \rho^{2}}{p-a(1-2 b \rho) \rho^{2}}
\end{aligned}
$$

avec $c_{\mathrm{p}_{0}}$ la chaleur spécifique d'un gaz parfait. 

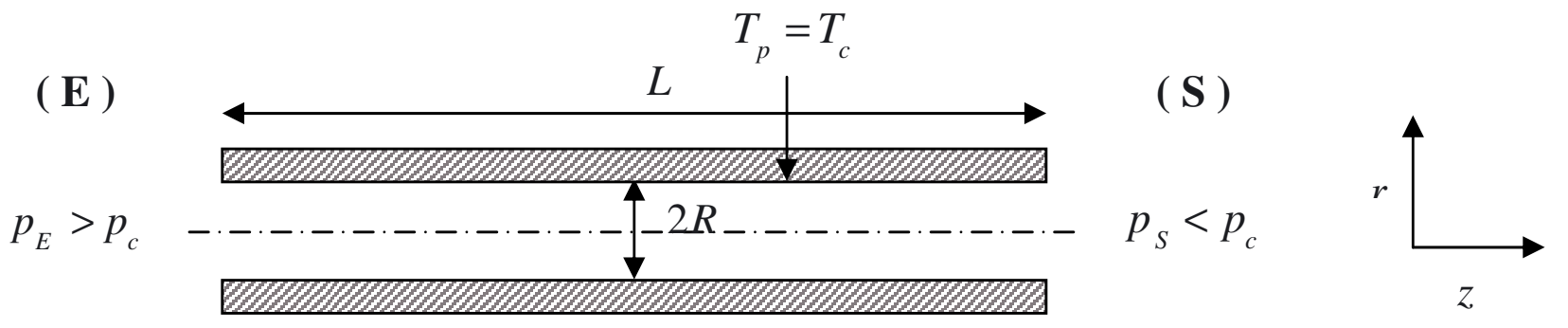

Fig. 1. Configuration de l'écoulement de filtration.

La conductivité thermique du fluide est une propriété qui n'obéit pas à la thermodynamique mais à la physique statistique. Dans le cadre de cette étude, on choisit une loi de comportement qui met en évidence la divergence au passage du voisinage du point critique [6-8] :

$$
k=k_{0}\left[\frac{\Lambda_{1}\left(T-T_{\mathrm{c}}\right)}{\left(p_{\mathrm{c}} / \rho_{\mathrm{c}} r_{v}\right)}+\frac{\Lambda_{2}\left(\rho-\rho_{\mathrm{c}}\right)}{\rho_{\mathrm{c}}}\right]^{-1 / 2}
$$

avec $k_{0}$ conductivité thermique d'un gaz parfait. $\Lambda_{1}$ et $\Lambda_{2}$ sont des constantes adimensionnelles d'ordre unité.

Dans le système (1), les conditions aux limites et de symétrie (en raison de la géométrie cylindrique du domaine d'écoulement) sont définies à partir des données du problème et des hypothèses. On a :

$$
\begin{array}{rlrl}
p(r ; 0) & =p_{\mathrm{E}}>p_{\mathrm{c}} & p(r ; L)=p_{\mathrm{S}}<p_{\mathrm{c}} \\
u(R ; z)=v(R ; z)=0 & T(R ; z)=T_{\mathrm{c}} \\
\frac{\partial u}{\partial r}(0 ; z)=\frac{\partial v}{\partial r}(0 ; z)=0 & \frac{\partial T}{\partial r}(0 ; z)=0
\end{array}
$$

\section{Première approche}

\section{1 Étude phénoménologique}

On définit dans le cadre de l'analyse phénoménologique du problème de filtration des variables sans dimension obtenues par un choix d'échelles approprié, qu'on écrit sous cette forme :

$$
\begin{aligned}
\frac{r}{R}=\bar{r} & \frac{z}{L}=\bar{z} & \frac{v}{\varepsilon U_{0}}=\bar{v} & \frac{u}{U_{0}}=\bar{u} \\
\frac{p}{p_{\mathrm{c}}}=\bar{p} & \frac{\rho}{\rho_{\mathrm{c}}}=\bar{\rho} & \frac{T}{\left(p_{\mathrm{c}} / \rho_{\mathrm{c}} r_{v}\right)}=\bar{T} & \frac{k}{k_{0}}=\bar{k} \\
\frac{c_{\mathrm{p}}}{c_{\mathrm{p} 0}}=\bar{c}_{\mathrm{p}} & \frac{\beta}{\beta_{0}}=\bar{\beta} & \frac{\mu}{\mu_{0}}=1 & \frac{\lambda}{\lambda_{0}}=1
\end{aligned}
$$

tel que : $\lambda_{0}+\frac{2}{3} \mu_{0}=0 \quad$ (hypothèse de Stokes).

On prend pour $U_{0}$ l'échelle de vitesse de Poiseuille :

$$
U_{0}=\frac{p_{\mathrm{c}} R^{2}}{4 \mu_{0} L}
$$

Le nombre sans dimension qui compare les effets visqueux et les effets dynamiques fait intervenir le facteur géométrique $\varepsilon$. Il a pour expression :

$$
R e_{\mathrm{R}} \varepsilon=\frac{\rho_{\mathrm{c}} U_{0} R^{2}}{\mu_{0} L} \quad \text { avec }: \quad R e_{\mathrm{R}}=\frac{\rho_{\mathrm{c}} U_{0} R}{\mu_{0}}
$$

$k_{0}, c_{\mathrm{p}_{0}}, \beta_{0}, \mu_{0}$ et $\lambda_{0}$ désignent respectivement les échelles de référence de la conductivité thermique, de la capacité calorifique à pression constante, du coefficient de dilatation, de la viscosité dynamique et volumique dans le cas d'un gaz parfait à une température $T_{0}$ (pour l'hydrogène on a choisi : $T_{0}=300 \mathrm{~K}$ ).

De ces quantités, le nombre de Prandtl $P r_{0}$ et l'indice adiabatique $\gamma_{0}$ s'expriment respectivement :

$$
P r_{0}=\frac{\mu_{0} c_{\mathrm{p}_{0}}}{k_{0}}=O(1) \quad \gamma_{0}=\frac{c_{\mathrm{p}_{0}}}{c_{v_{0}}}=O(1)
$$

Les coefficients de viscosité dynamique et volumique du fluide sont considérés constants [9].

En choisissant pour les variations de vitesse $U_{0}$, l'échelle de Poiseuille due à une différence de pression de l'ordre de la pression critique $\left(p_{\mathrm{c}}\right)$, on considère une situation de régime établi (où l'échelle de longueur est très supérieure à la longueur d'établissement $L \gg L_{\mathrm{e}}[5]$ ) qui se traduit par la condition sur la donnée suivante : $R e_{\mathrm{R}} \varepsilon \ll 1$.

A titre d'exemple, pour l'hydrogène $\left(p_{\mathrm{c}}\right.$ et $\rho_{\mathrm{c}}$ coordonnées thermodynamiques critiques réelles) :

$$
\begin{aligned}
& p_{\mathrm{c}}=1,28 \times 10^{6} \mathrm{~Pa}, \rho_{\mathrm{c}}=31,36 \mathrm{~kg} \cdot \mathrm{m}^{-3}, T_{0}=300 \mathrm{~K}, \\
& \mu_{0}=8,14 \times 10^{-6} \mathrm{~kg} \cdot \mathrm{m}^{-1} \cdot \mathrm{s}^{-1}
\end{aligned}
$$

dans un capillaire de dimensions : $L=0,3 \mathrm{~m} R=10^{-5} \mathrm{~m}$

On obtient : $R e_{\mathrm{R}} \varepsilon=0,017$

\section{2 Écoulement de base}

En se limitant pour le moment aux termes d'ordre unité, les grandeurs de l'écoulement $\bar{v}, \bar{u}, \bar{p}, \bar{\rho}$ et $\bar{T}$ sont recherchées sous la forme de développements écrits de la sorte :

$$
\begin{array}{ll}
\bar{v}=\bar{v}_{0}(\bar{r} ; \bar{z})+o(1) & \bar{u}=\bar{u}_{0}(\bar{r} ; \bar{z})+o(1) \\
\bar{p}=\bar{p}_{0}(\bar{r} ; \bar{z})+o(1) & \bar{T}=\bar{T}_{0}(\bar{r} ; \bar{z})+o(1) \\
\bar{\rho}=\bar{\rho}_{0}(\bar{r} ; \bar{z})+o(1) &
\end{array}
$$


En reportant ces quantités (6) dans les équations de la dynamique, la thermique et le modèle de Van der Waals (1), on obtient en première approximation $(\varepsilon \rightarrow 0$, $\left.R e_{\mathrm{R}} \varepsilon \rightarrow 0\right):$

$$
\begin{aligned}
& \frac{1}{\bar{r}} \frac{\partial}{\partial \bar{r}}\left(\bar{r} \bar{\rho}_{0} \bar{v}_{0}\right)+\frac{\partial}{\partial \bar{z}}\left(\bar{\rho}_{0} \bar{u}_{0}\right)=0 \\
& -4 \frac{\mathrm{d} \bar{p}_{0}}{\mathrm{~d} \bar{z}}+\frac{1}{\bar{r}} \frac{\partial}{\partial \bar{r}}\left(\bar{r} \frac{\partial \bar{u}_{0}}{\partial \bar{r}}\right)=0 \\
& \frac{1}{\bar{r}} \frac{\partial}{\partial \bar{r}}\left(\bar{k}_{0} \bar{r} \frac{\partial \bar{T}_{0}}{\partial \bar{r}}\right)=0 \\
& \bar{p}_{0}=\frac{3 \bar{\rho}_{0} \bar{T}_{0}}{3-\bar{\rho}_{0}}-3 \bar{\rho}_{0}^{2}
\end{aligned}
$$

avec les conditions aux limites et de symétrie suivantes :

$$
\begin{array}{lr}
\bar{u}_{0}(1 ; \bar{z})=\bar{v}_{0}(1 ; \bar{z})=0 & \bar{T}_{0}(1 ; \bar{z})=\frac{8}{3} \\
\bar{p}_{0}(0)=\bar{p}_{\mathrm{E}} & \bar{p}_{0}(1)=\bar{p}_{\mathrm{S}} \\
\frac{\partial \bar{u}_{0}}{\partial \bar{r}}(0 ; \bar{z})=0 & \frac{\partial \bar{T}_{0}}{\partial \bar{r}}(0 ; \bar{z})=0
\end{array}
$$

Dans l'équation thermique écrite en (7), on trouve que la température du fluide suit celle de la paroi du capillaire au premier ordre (température critique) :

$$
\bar{T}_{0}(\bar{r} ; \bar{z})=\frac{8}{3}
$$

À partir du champ de Poiseuille, obtenu par la dynamique en $(7)$ :

$$
\bar{u}_{0}(\bar{r}, \bar{z})=\frac{\mathrm{d} \bar{p}_{0}}{\mathrm{~d} \bar{z}}\left(\bar{r}^{2}-1\right)
$$

le débit massique s'exprime en fonction du gradient de pression :

$$
\begin{gathered}
\bar{Q}_{0}=-\frac{\bar{\rho}_{0}}{4} \frac{\mathrm{d} \bar{p}_{0}}{\mathrm{~d} \bar{z}} \quad \text { avec }: \quad \bar{Q}_{0}=Q / \Delta Q_{0} \\
\text { et } \Delta Q_{0}=\pi R^{4} p_{\mathrm{c}} \rho_{\mathrm{c}} / 4 \mu_{0} L
\end{gathered}
$$

De cette forme différentielle ordinaire (en $\bar{z}=0$; $\left.\bar{\rho}_{\mathrm{E}}\left(\bar{p}_{\mathrm{E}}, \bar{T}_{0}\right)\right)$, on obtient l'évolution de la masse volumique de l'écoulement de filtration selon la forme inverse suivante :

$$
\bar{z}\left(\bar{\rho}_{0}\right)=\frac{1}{\bar{Q}_{0}}\left[\frac{\bar{\rho}_{0}^{3}}{2}-\frac{18}{\left(3-\bar{\rho}_{0}\right)}-6 \ln \left(3-\bar{\rho}_{0}\right)+\bar{C}_{0}\right]
$$

avec : $\bar{C}_{0}=\bar{C}_{0}\left(\bar{\rho}_{\mathrm{E}}\right)$.

Le débit massique du fluide qui est dû à une différence de pression $\bar{p}_{\mathrm{E}}-\bar{p}_{\mathrm{S}}$ entre l'entrée et la sortie du conduit a pour expression dans ce cas $: \mathrm{w}$

$$
\bar{Q}_{0}=\frac{\bar{\rho}_{\mathrm{S}}^{3}-\bar{\rho}_{\mathrm{E}}^{3}}{2}+\frac{18\left(\bar{\rho}_{\mathrm{E}}-\bar{\rho}_{\mathrm{S}}\right)}{\left(3-\bar{\rho}_{\mathrm{S}}\right)\left(3-\bar{\rho}_{\mathrm{E}}\right)}+6 \ln \left(\frac{3-\bar{\rho}_{\mathrm{E}}}{3-\bar{\rho}_{\mathrm{S}}}\right)
$$

Par une simple manipulation de l'expression différentielle du débit $\left(\bar{Q}_{0}=-\left(\bar{\rho}_{0} / 4\right)\left(\mathrm{d} \bar{p}_{0} / \mathrm{d} \bar{z}\right)\right)$, le champ de pression dans le capillaire a pour évolution :

$$
\bar{p}_{0}(\bar{z})=\frac{3}{3-\bar{\rho}_{0}}\left[\left(\bar{\rho}_{0}-3\right)^{2}\left(\bar{\rho}_{0}+3\right)+8\right]+\bar{C}_{1}
$$

avec : $\bar{C}_{1}=\bar{C}_{1}\left(\bar{\rho}_{\mathrm{E}}\right)$.
Bien que la première approximation de Navier Stokes ne signale aucune singularité au passage du point critique $\left(\bar{z}=\bar{z}_{\mathrm{c}}\right.$ à $\left.\bar{\rho}_{0}=1\right)$, les propriétés sans dimension $\bar{k}_{0}, \bar{\beta}_{0}$ et $\bar{c}_{\mathrm{p}_{0}}$ à l'ordre « un » divergent à cette échelle [5].

\subsection{Perturbation singulière due à la dilatation}

L'influence du comportement singulier de ces propriétés physiques sur l'écoulement est recherchée au second ordre par le développement de ces diverses quantités du fluide :

$$
\begin{aligned}
& \bar{v}=\delta_{v_{1}} \bar{v}_{1}(\bar{r} ; \bar{z})+o\left(\delta_{v_{1}}\right) \\
& \bar{u}=\bar{u}_{0}(\bar{r} ; \bar{z})+\delta_{u_{1}} \bar{u}_{1}(\bar{r} ; \bar{z})+o\left(\delta_{u_{1}}\right) \\
& \bar{p}=\bar{p}_{0}(\bar{z})+\delta_{p_{1}} \bar{p}_{1}(\bar{r} ; \bar{z})+o\left(\delta_{p_{1}}\right) \\
& \bar{\rho}=\bar{\rho}_{0}(\bar{z})+\delta_{\rho_{1}} \bar{\rho}_{1}(\bar{r} ; \bar{z}) \\
& \bar{T}=\bar{T}_{0}(\bar{r} ; \bar{z})+\delta_{T_{1}} \bar{T}_{1}(\bar{r} ; \bar{z})+o\left(\delta_{T_{1}}\right)+o\left(\delta_{\rho_{1}}\right)
\end{aligned}
$$

Les fonctions de jauge $\delta_{v_{1}}, \delta_{u_{1}}, \delta_{p_{1}}, \delta_{\rho_{1}}$ et $\delta_{T_{1}}$ sont déterminées par moindre dégénérescence $[5,10]\left(\delta_{T_{1}}=\right.$ $\left.\delta_{p_{1}}=\delta_{u_{1}}=\delta_{\rho_{1}}=\delta_{v_{1}}=\varepsilon R e_{\mathrm{R}}\right)$.

En seconde approximation $\left(\varepsilon \rightarrow 0, R e_{\mathrm{R}} \varepsilon \rightarrow 0\right)$, on obtient :

$$
\begin{aligned}
& \frac{1}{\bar{r}} \frac{\partial}{\partial \bar{r}}\left(\bar{r} \bar{\rho}_{0} \bar{v}_{1}\right)+\frac{\partial}{\partial \bar{z}}\left(\bar{\rho}_{1} \bar{u}_{0}\right)+\frac{\partial}{\partial \bar{z}}\left(\bar{\rho}_{0} \bar{u}_{1}\right)=0 \\
& \bar{\rho}_{0} \bar{u}_{0} \frac{\partial \bar{u}_{0}}{\partial \bar{z}}=-4 \frac{\mathrm{d} \bar{p}_{1}}{\mathrm{~d} \bar{z}}+\frac{1}{\bar{r}} \frac{\partial}{\partial \bar{r}}\left(\bar{r} \frac{\partial \bar{u}_{1}}{\partial \bar{r}}\right) \\
& \frac{\Lambda}{P r_{0}} \frac{1}{\left|\bar{\rho}_{0}-1\right|^{1 / 2}} \frac{1}{\bar{r}} \frac{\partial}{\partial \bar{r}}\left(\bar{r} \frac{\partial \bar{T}_{1}}{\partial \bar{r}}\right)=-\left(\frac{\gamma_{0}-1}{\gamma_{0}}\right) \\
& \times\left[\frac{8}{3} \bar{\beta}_{0} \bar{u}_{0} \frac{\mathrm{d} \bar{p}_{0}}{\mathrm{~d} \bar{z}}+\frac{1}{4}\left(\frac{\partial \bar{u}_{0}}{\partial \bar{r}}\right)^{2}\right] \\
& \bar{p}_{1}=\frac{24 \bar{\rho}_{1}}{\left(3-\bar{\rho}_{0}\right)^{2}}-6 \bar{\rho}_{0} \bar{\rho}_{1}+\frac{3 \bar{\rho}_{0} \bar{T}_{1}}{3-\bar{\rho}_{0}}
\end{aligned}
$$

avec comme conditions aux limites et de symétrie :

$$
\begin{aligned}
& \bar{u}_{1}(1 ; \bar{z})=\bar{v}_{1}(1 ; \bar{z})=0 \quad \bar{p}_{1}(0)=0 \\
& \bar{p}_{1}(1)=0 \quad \bar{T}_{1}(1 ; \bar{z})=0 \\
& \frac{\partial \bar{u}_{1}}{\partial \bar{r}}(0 ; \bar{z})=0 \quad \frac{\partial \bar{T}_{1}}{\partial \bar{r}}(0 ; \bar{z})=0
\end{aligned}
$$

Ce modèle en seconde approximation (14), (15) a été obtenu par une analyse phénoménologique du problème qui nous a permis de maintenir les termes dominants à cet ordre. La correction des équations de Navier Stokes (1) a bien mis en évidence l'influence des propriétés physiques sur le comportement de l'écoulement. On le voit clairement dans l'équation de la chaleur (14) à travers les termes de diffusion et de production (forces de pression). 


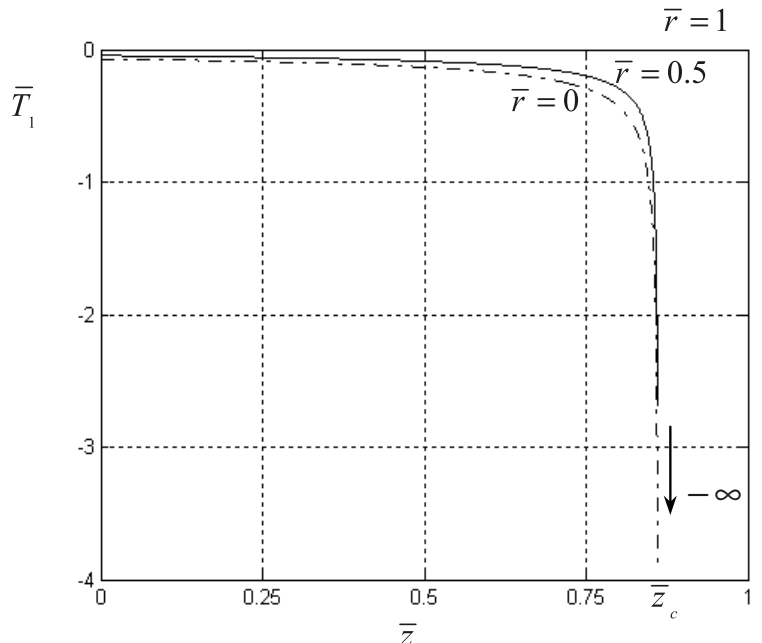

Fig. 2. Évolution de la variation de la température dans le capillaire.

À cette étape de l'étude, on s'est limité seulement à exprimer l'évolution de la température du fluide au voisinage du point critique :

$$
\begin{array}{r}
\bar{T}_{1}(\bar{r}, \bar{z})=-\Pi_{0} \bar{Q}_{0}^{2} \frac{\sqrt{\bar{\rho}_{0}-1}}{\bar{\rho}_{0}^{2}}\left[\bar{r}^{2}+1+\frac{8}{3} \bar{\beta}_{0}\left(\bar{r}^{2}-3\right)\right] \\
\times\left(\bar{r}^{2}-1\right)
\end{array}
$$

avec :

$$
\begin{aligned}
\bar{\beta}_{0} & =\frac{\bar{\rho}_{0}}{2 \bar{\rho}_{0}^{3}-3 \bar{\rho}_{0}^{2}+\bar{p}_{0}} \quad \Pi_{0}=\frac{\left(\gamma_{0}-1\right) \operatorname{Pr}_{0}}{\Lambda \gamma_{0}}=O(1) \\
\Lambda & =\Lambda_{2}^{1 / 2}=O(1)
\end{aligned}
$$

Sous l'effet de la dilatation, le refroidissement de l'écoulement (Fig. 2) s'effectue de la paroi vers le centre (axe de symétrie) et dans le sens du mouvement. Il devient singulier à la position $\bar{z}_{\mathrm{c}}$ (état thermodynamique critique). Cette chute de température s'explique par un refroidissement dans le cas d'une détente non isentropique, comme on peut le voir par la quantité de chaleur $\bar{q}_{r}$ perdue transversalement vers l'extérieur à travers la paroi (Fig. 3). Cette singularité qui est d'origine thermique se transmet aux autres grandeurs physiques $\left(\bar{v}_{1}, \bar{u}_{1}, \bar{p}_{1}\right.$ et $\left.\bar{\rho}_{1}\right)$ du fluide [5].

Par ailleurs, la correction apportée au débit massique adimensionnel de base $\bar{Q}_{0}$ définie par :

$$
\begin{aligned}
& \bar{Q}_{1}=\int_{0}^{1}\left(\bar{\rho}_{0} \bar{u}_{1}+\bar{u}_{0} \bar{\rho}_{1}\right) \bar{r} \mathrm{~d} \bar{r} \\
& \text { où : } \bar{Q}=\bar{Q}_{0}+\varepsilon R e_{\mathrm{R}} \bar{Q}_{1}+o\left(\varepsilon R e_{\mathrm{R}}\right)
\end{aligned}
$$

est inconnue à ce stade de l'étude (tranche : $\bar{z} \leq \bar{z}_{\mathrm{c}}$ ) en raison d'un manque de données dû à la présence de la zone singulière $\left(\bar{z}=\bar{z}_{\mathrm{c}}\right)$. On y reviendra ultérieurement.

Dans cette région critique, les grandeurs du fluide ne sont donc plus uniformément valables car les échelles des

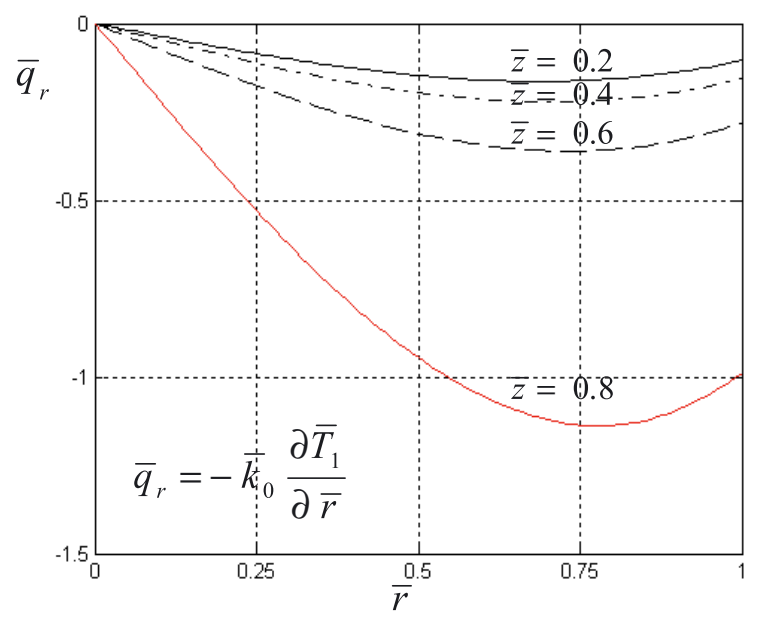

Fig. 3. Évolution du flux de chaleur radial pour différentes sections.

corrections changent d'ordre de grandeur (Fig. 2). Un nouveau dimensionnement s'impose au voisinage de $\bar{z}_{\mathrm{c}}$ pour décrire une nouvelle variation caractérisée par l'existence d'une zone de transition (couche limite libre) [11]. La continuité des différents comportements est assurée par la technique des développements asymptotiques raccordés [10].

\section{Zone de filtration à forte dilatation}

\subsection{Modélisation de la région intérieure}

L'examen du fluide dans la couche limite d'épaisseur $\eta$ («1, à déterminer) s'effectue de la même façon que dans la première approche. Comme on est au voisinage du point critique, les comportements de raccord de la région amont [5] nous permettent d'obtenir les nouvelles variables intérieures sans dimension, définies de la façon suivante :

$$
\begin{aligned}
& \frac{r}{R}=\bar{r} \quad \frac{z}{L}=\bar{z}_{\mathrm{c}}+\eta \tilde{z} \quad \frac{v}{U_{0}}=\frac{\varepsilon}{\eta} \tilde{v}_{1} \\
& \frac{u}{U_{0}}=\tilde{u}_{0}(\bar{r})+\eta^{1 / 3} \tilde{u}_{1} \quad \frac{p}{p_{c}}=1+\eta \tilde{p}_{1} \quad \frac{\rho}{\rho_{c}}=1+\eta^{1 / 3} \tilde{\rho}_{1} \\
& \frac{T}{\left(p_{\mathrm{c}} / \rho_{\mathrm{c}} r_{\mathrm{v}}\right.}=\frac{8}{3}+\eta \tilde{T}_{1} \quad \frac{k}{k_{0}}=\tilde{k}_{0} \quad \frac{c_{\mathrm{p}}}{c_{\mathrm{p}_{0}}}=\tilde{c}_{\mathrm{p}_{0}} \frac{\mu}{\mu_{0}}=1 \\
& \frac{\lambda}{\lambda_{0}}=1 \quad \text { avec }: \tilde{u}_{0}(\bar{r})=\bar{u}_{0}(\bar{r})
\end{aligned}
$$

L'échelle de vitesse débitante de Poiseuille $U_{0}$ a été maintenue dans cette zone en raison de la condition de raccord. On a maintenu également le nombre de Reynolds $R e_{\mathrm{R}}$, de Prandtl $P r_{0}$ et le rapport des chaleurs spécifiques $\gamma_{0}$. Par contre, un nouveau nombre sans dimension apparait dans cette nouvelle description qui est le nombre d'Eckert :

$$
E_{0}=\frac{U_{0}^{2}}{c_{\mathrm{p}_{0}} T_{\mathrm{c}}} \quad\left(E_{0}=3,5 \times 10^{-4} \text { pour l'hydrogène }\right)
$$


Le raccord de la température physique entre la partie amont et la région critique est donné par :

$$
\begin{aligned}
T_{\mathrm{c}}\left[\frac{8}{3}+\frac{\varepsilon R e_{\mathrm{R}}}{\eta^{1 / 2}} \lim _{\bar{z} \rightarrow \bar{z}_{\mathrm{c}}^{-}} \bar{T}_{1}(\bar{r} ; \bar{z})\right] & = \\
T_{\mathrm{c}}\left[\frac{8}{3}+\eta\right. & \left.\lim _{\bar{z} \rightarrow-\infty} \tilde{T}_{1}(\bar{r} ; \tilde{z})\right]
\end{aligned}
$$

On en déduit l'épaisseur de la couche limite : $\eta=\left(\varepsilon R e_{\mathrm{R}}\right)^{2 / 3}$.

À partir de là, les développements des variables sans dimension définies ci-dessus ont été reportés dans les équations de Navier Stokes et la loi d'état de Van der Waals pour finalement nous donner en première approximation $\left(\varepsilon \rightarrow 0, R e_{\mathrm{R}} \varepsilon \rightarrow 0\right)$ :

$$
\begin{aligned}
& \frac{1}{\bar{r}} \frac{\partial}{\partial \bar{r}}\left(\bar{r} \tilde{v}_{1}\right)+\tilde{u}_{0} \frac{\partial \tilde{\rho}_{1}}{\partial \tilde{z}}+\frac{\partial \tilde{u}_{1}}{\partial \tilde{z}}=0 \\
& -4 \frac{\mathrm{d} \tilde{p}_{1}}{\mathrm{~d} \tilde{z}}+\frac{1}{\bar{r}} \frac{\mathrm{d}}{\mathrm{d} \bar{r}}\left(\bar{r} \frac{\mathrm{d} \tilde{u}_{0}}{\mathrm{~d} \bar{r}}\right)=0 \\
& \tilde{c}_{\mathrm{p}_{0}} \tilde{u}_{0} \frac{\partial \tilde{T}_{1}}{\partial \tilde{z}}-\frac{1}{\operatorname{Pr}_{0}} \frac{1}{\bar{r}} \frac{\partial}{\partial \bar{r}}\left(\tilde{k}_{0} \bar{r} \frac{\partial \tilde{T}_{1}}{\partial \bar{r}}\right)=\frac{8}{3}\left(\frac{\gamma_{0}-1}{\gamma_{0}}\right) \tilde{\beta}_{0} \tilde{u}_{0} \frac{\mathrm{d} \tilde{p}_{1}}{\mathrm{~d} \tilde{z}} \\
& \frac{2}{3} \tilde{p}_{1}=\tilde{\rho}_{1}^{3}+\tilde{T}_{1}
\end{aligned}
$$

Afin de fermer ce système (17), on a rajouté deux équations supplémentaires (18) dont l'une est intégrodifférentielle. Celle-ci a été obtenue à partir du développement de l'équation de quantité de mouvement au deuxième ordre combinée à l'expression de la correction du débit massique dans la région intérieure, d'où :

$$
\begin{aligned}
& 4 \frac{\mathrm{d} \tilde{p}_{2}}{\mathrm{~d} \tilde{z}}-\frac{1}{\bar{r}} \frac{\partial}{\partial \bar{r}}\left(\bar{r} \frac{\partial \tilde{u}_{1}}{\partial \bar{r}}\right)=0 \\
& \frac{\mathrm{d} \tilde{p}_{2}}{\mathrm{~d} \tilde{z}}=16 \bar{Q}_{0} \int_{0}^{1} \tilde{\rho}_{1}\left(\bar{r}-\bar{r}^{3}\right) \mathrm{d} \bar{r}-4 \tilde{Q}_{1}
\end{aligned}
$$

avec : $\tilde{Q}_{1}=\int_{0}^{1}\left(\tilde{u}_{1}+\tilde{u}_{0} \tilde{\rho}_{1}\right) \bar{r} \mathrm{~d} \bar{r}$ où :

$\tilde{Q}=\bar{Q}_{0}+\left(\varepsilon R e_{\mathrm{R}}\right)^{2 / 9} \tilde{Q}_{1}+o\left[\left(\varepsilon R e_{\mathrm{R}}\right)^{2 / 9}\right]$.

Au final, ce modèle (17), (18) doit satisfaire les conditions aux limites sur les parois et sur l'axe :

$$
\begin{array}{lrl}
\tilde{v}_{1}(1 ; \tilde{z})=\tilde{u}_{1}(1 ; \tilde{z})=0 & \tilde{T}_{1}(1 ; \tilde{z})=0 \\
\frac{\partial \tilde{u}_{1}}{\partial \bar{r}}(0 ; \tilde{z})=0 & \frac{\partial \tilde{T}_{1}}{\partial \bar{r}}(0 ; \tilde{z})=0
\end{array}
$$

ainsi que les conditions de raccord [5] :

$$
\begin{aligned}
\tilde{p}_{1}(-\infty) & =\frac{3}{2} \tilde{F}_{0}^{3}(\tilde{z}) \\
\tilde{u}_{1}(\bar{r} ;-\infty) & =4 \bar{Q}_{0}\left[\tilde{F}_{0}(\tilde{z})+\frac{44 \Pi_{0} \bar{Q}_{0}^{2}}{81 \tilde{F}_{0}^{7 / 2}(\tilde{z})}\right]\left(\bar{r}^{2}-1\right) \\
\tilde{T}_{1}(\bar{r} ;-\infty) & =-\frac{8 \Pi_{0} \bar{Q}_{0}^{2}\left(\bar{r}^{2}-1\right)\left(\bar{r}^{2}-3\right)}{9 \tilde{F}_{0}^{3 / 2}(\tilde{z})} \\
\tilde{p}_{2}(-\infty) & =-\frac{9}{8} \tilde{F}_{0}^{4}(\tilde{z})+\frac{44 \Pi_{0} \bar{Q}_{0}^{2}}{9 \tilde{F}_{0}^{1 / 2}(\tilde{z})} \\
\tilde{\rho}_{1}(\bar{r} ;-\infty) & =\tilde{F}_{0}(\tilde{z})+\frac{8 \Pi_{0} \bar{Q}_{0}^{2}\left(\bar{r}^{2}-1\right)\left(\bar{r}^{2}-3\right)}{27 \tilde{F}_{0}^{7 / 2}(\tilde{z})} \\
\text { avec }: \quad\left(\frac{8 \bar{Q}_{0} \tilde{z}}{3}\right)^{1 / 3} &
\end{aligned}
$$

Les paramètres physiques du fluide dans cette région ont pour comportement [5] :

$$
\tilde{k}_{0} \sim \Lambda\left|\tilde{F}_{0}(\tilde{z})\right|^{-1 / 2}, \tilde{c}_{\mathrm{p}_{0}} \sim \frac{4\left(\gamma_{0}-1\right)}{3 \gamma_{0}} \tilde{F}_{0}^{-2}(\tilde{z}), \tilde{\beta}_{0} \sim \frac{1}{3} \tilde{F}_{0}^{-2}(\tilde{z})
$$

\section{2 Écoulement critique}

La modélisation de la couche limite présentée dans le paragraphe précédent nous a permis de mettre en évidence l'existence d'un terme de convection thermique dans cette région qui s'ajoute à la conduction radiale afin d'assurer le transport de chaleur. Dans cette description, on a observé également la présence d'un couplage thermo-dynamique favorisé par la forte dilatation du milieu comme le montre la loi d'état.

Le comportement local de l'écoulement au sein de la zone de transition a été obtenu par un traitement numérique des équations de la mécanique des fluides $(17,18)$. On s'assure de la validité de la représentation globale en vérifiant la compatibilité du modèle numérique de la couche limite avec le modèle analytique au niveau du raccord. Pour cela, on a fait appel à une technique dite « variable like expansion » (VLE) qui permet de rechercher les solutions à l'infini [12]. Cette méthode (VLE) a été utilisée deux fois dans cette région, car cette dernière doit être raccordée à l'entrée (infini amont) et à la sortie subcritique (infini aval). Le raccord physique des différentes grandeurs du fluide $(\tilde{v}, \tilde{u}, \tilde{p}, \tilde{\rho}, \tilde{T})$ a permis parallèlement de déduire que la correction du débit massique $\tilde{Q}_{1}$ à l'échelle $\left(\varepsilon R e_{\mathrm{R}}\right)^{2 / 9}$ est nulle [5].

$\mathrm{Au}$ final, la résolution numérique de ce modèle par un algorithme en différence finie (avec : $\Delta \tilde{z}=10^{-2}, \Delta \bar{r}=$ $10^{-2}, \tilde{Q}_{0}=\bar{Q}_{0}=0,2, \Pi_{0}=1$ ) a montré un comportement régulier de l'écoulement dans cette zone comme on peut le constater à travers l'évolution de la température (Fig. 4).

Le mécanisme de transport thermique, assuré conjointement par la convection forcée et la conduction, régule l'évolution globale de l'écoulement $(\tilde{v}, \tilde{u}, \tilde{p}, \tilde{\rho}, \tilde{T})$ dans cette région hypercompressible $(\tilde{z}=0)$. Sous le contrôle 


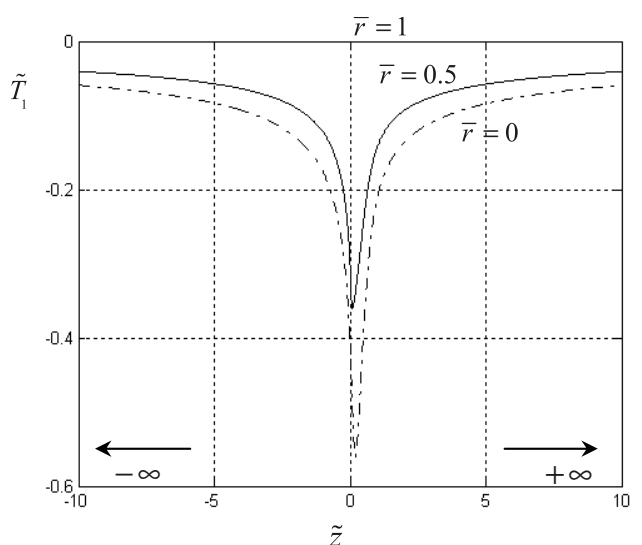

Fig. 4. Évolution de la variation de la température intérieure dans la couche limite libre.

de la dilatation massique, le milieu fluide se refroidit certes mais de manière régulière (Fig. 4) pour ensuite se réchauffer jusqu'à ce qu'il retrouve la température d'entrée de la couche limite (symétrie à l'infini).

\section{Région extérieure aval}

\subsection{Evolution du fluide vers la sortie du capillaire}

En dernière partie dans la zone extérieure aval, on a étudié l'influence de la traversée du point critique sur le comportement du fluide proche de la sortie. En se basant sur la première approche qui a bien montré une évolution régulière de l'écoulement de base (7) tout le long du conduit, l'évaluation de cet accident de filtration ne peut être faite que par l'examen de la deuxième approximation $\left(\bar{v}_{1}, \bar{u}_{1}, \bar{p}_{1}, \bar{\rho}_{1}, \bar{T}_{1}\right)$. Par moindre dégénérescence $(\varepsilon \rightarrow 0$, $\left.R e_{\mathrm{R}} \varepsilon \rightarrow 0\right)$, on obtient :

$$
\begin{aligned}
& \frac{1}{\bar{r}} \frac{\partial}{\partial \bar{r}}\left(\bar{r} \bar{\rho}_{0} \bar{v}_{1}\right)+\frac{\partial}{\partial \bar{z}}\left(\bar{\rho}_{1} \bar{u}_{0}\right)+\frac{\partial}{\partial \bar{z}}\left(\bar{\rho}_{0} \bar{u}_{1}\right)=0 \\
& \bar{\rho}_{0} \bar{u}_{0} \frac{\partial \bar{u}_{0}}{\partial \bar{z}}=-4 \frac{\mathrm{d} \bar{p}_{1}}{\mathrm{~d} \bar{z}}+\frac{1}{\bar{r}} \frac{\partial}{\partial \bar{r}}\left(\bar{r} \frac{\partial \bar{u}_{1}}{\partial \bar{r}}\right) \\
& \frac{\Lambda}{P r_{0}} \frac{1}{\left(1-\bar{\rho}_{0}\right)^{1 / 2}} \frac{1}{\bar{r}} \frac{\partial}{\partial \bar{r}}\left(\bar{r} \frac{\partial \bar{T}_{1}}{\partial \bar{r}}\right)=-\left(\frac{\gamma_{0}-1}{\gamma_{0}}\right) \\
& \times\left[\frac{8}{3} \bar{\beta}_{0} \bar{u}_{0} \frac{\mathrm{d} \bar{p}_{0}}{\mathrm{~d} \bar{z}}+\frac{1}{4}\left(\frac{\partial \bar{u}_{0}}{\partial \bar{r}}\right)^{2}\right] \\
& \bar{p}_{1}=\frac{24 \bar{\rho}_{1}}{\left(3-\bar{\rho}_{0}\right)^{2}}-6 \bar{\rho}_{0} \bar{\rho}_{1}+\frac{3 \bar{\rho}_{0} \bar{T}_{1}}{3-\bar{\rho}_{0}}
\end{aligned}
$$

avec comme conditions aux limites [5] :

$$
\begin{aligned}
\bar{u}_{1}(1 ; \bar{z}) & =\bar{v}_{1}(1 ; \bar{z})=0 \\
\bar{p}_{1}\left(\bar{z}_{\mathrm{c}}^{+}\right) & =-\frac{44 \Pi_{0} \bar{Q}_{0}^{2}}{9}\left(\frac{8 \bar{Q}_{0}}{3}\right)^{-1 / 6}\left(\bar{z}-\bar{z}_{c}\right)^{-1 / 6} \\
\bar{T}_{1}(1 ; \bar{z}) & =0
\end{aligned}
$$

et de symétrie :

$$
\frac{\partial \bar{u}_{1}}{\partial \bar{r}}(0 ; \bar{z})=0 \quad \frac{\partial \bar{T}_{1}}{\partial \bar{r}}(0 ; \bar{z})=0
$$

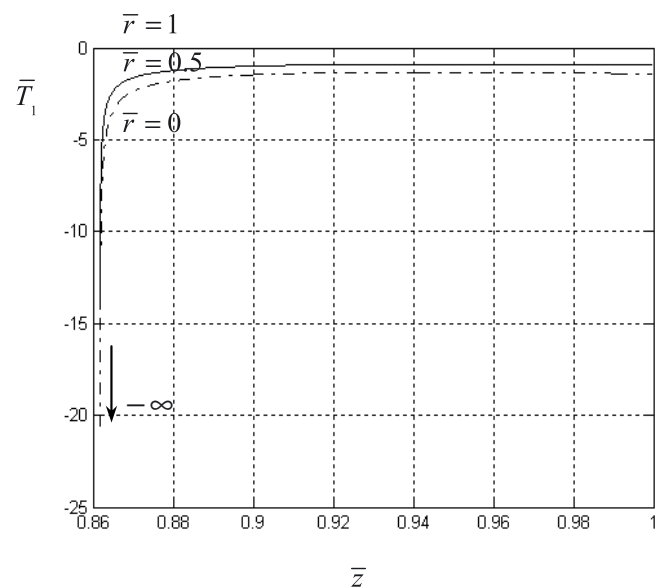

Fig. 5. Évolution de la variation de la température extérieure en aval du point critique.

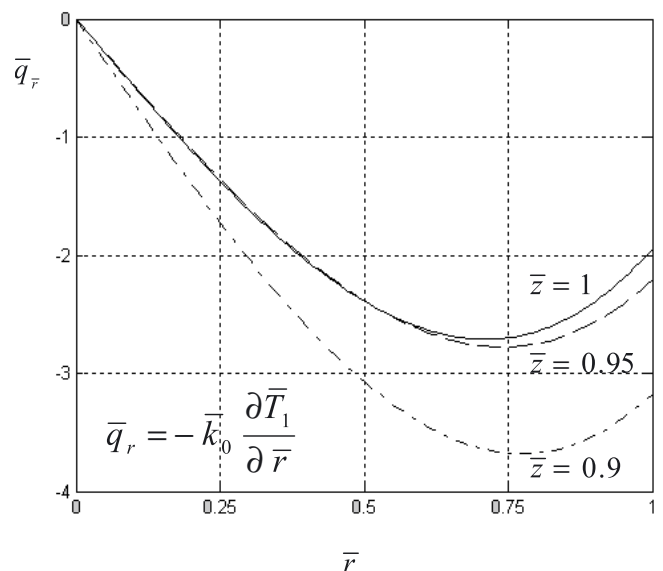

Fig. 6. Flux de chaleur radial pour des sections en aval de la couche limite.

Malgré la présence d'une couche limite au passage du point critique, les échelles de variation des grandeurs $(\bar{v}, \bar{u}, \bar{p}, \bar{\rho}, \bar{T})$ en amont se conservent en aval $\left(\varepsilon R e_{\mathrm{R}}\right)$. Ce résultat est bien évidement la conséquence d'une phénoménologie globalement identique à l'extérieur comme on peut le voir d'après le modèle (21)-(23).

Dans l'équation thermique ci-dessus, le terme de convection a disparu et la conduction radiale contrôle seule à nouveau le transport de chaleur. Dans cette région, la température de l'écoulement a pour comportement (Fig. 5) :

$$
\begin{array}{r}
\bar{T}_{1}(\bar{r}, \bar{z})=-\Pi_{0} \bar{Q}_{0}^{2} \frac{\sqrt{1-\bar{\rho}_{0}}}{\bar{\rho}_{0}^{2}}\left[\bar{r}^{2}+1+\frac{8}{3} \bar{\beta}_{0}\left(\bar{r}^{2}-3\right)\right] \\
\times\left(\bar{r}^{2}-1\right)
\end{array}
$$

À la sortie de la zone de transition $\left(\bar{z}=\bar{z}_{\mathrm{c}}^{+}\right)$où la dilatabilité du milieu est décroissante, le fluide perd de moins en moins de l'énergie calorifique vers l'extérieur (Fig. 6). En fin de parcours, l'écoulement dans sa globalité retrouve le comportement de base $\left(\bar{z} \ll \bar{z}_{\mathrm{c}}\right)$.

Cette dernière description (21) a été à son tour validée par les raccords avec la région de transition [5], ce qui 


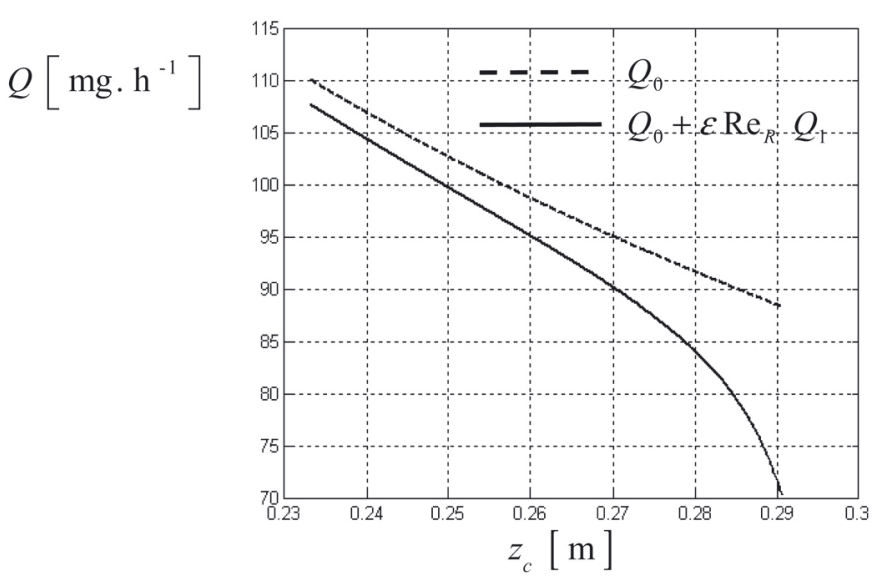

Fig. 7. Évolution du débit massique en fonction de la position du point critique.

nous a permis par la suite de déduire la correction du débit massique $\bar{Q}_{1}$ en fonction des données de sortie et la position de la couche limite :

$$
\begin{array}{r}
\bar{Q}_{1}=\frac{\bar{Q}_{0}^{2}}{4\left(1-\bar{z}_{\mathrm{c}}\right)}\left\{2 \ln \left(\bar{\rho}_{\mathrm{S}}\right)+\frac{\Pi_{0}}{3}\left[5 \sqrt{2} \arctan \left(\frac{\sqrt{1-\bar{\rho}_{\mathrm{S}}}}{\sqrt{2}}\right)\right.\right. \\
-\frac{11 \sqrt{3}}{9} \arctan \left(\frac{\sqrt{1-\bar{\rho}_{\mathrm{S}}}}{\sqrt{3}}\right)+3 \ln \left|\frac{1+\sqrt{1-\bar{\rho}_{\mathrm{S}}}}{-1+\sqrt{1-\bar{\rho}_{\mathrm{S}}}}\right| \\
\left.\left.-\frac{44}{3} \frac{1}{\sqrt{1-\bar{\rho}_{\mathrm{S}}}}\right]\right\}(25)
\end{array}
$$

\subsection{Effet bouchon}

L'étude phénoménologique de l'écoulement traversant le voisinage du point critique s'est donc scindée en trois parties. La cohérence de cette démarche physique qui a été validée par le raccord des trois régions du fluide a permis une meilleure estimation du débit massique qui tient compte de la présence de la zone de transition critique. Il a pour expression dimensionnelle (en $\left.\mathrm{mg} \cdot \mathrm{h}^{-1}\right)$ :

$$
Q=Q_{0}\left(p_{\mathrm{E}} ; p_{\mathrm{S}}\right)+\varepsilon R e_{\mathrm{R}} Q_{1}\left(p_{\mathrm{E}} ; p_{\mathrm{S}} ; z_{\mathrm{c}}\left(p_{\mathrm{E}} ; p_{\mathrm{S}}\right)\right)
$$

L'impact réel de cette couche limite sur la dynamique de l'écoulement entre l'entrée et la sortie du capillaire a été représenté graphiquement (Fig. 7) dans le cas de l'hydrogène avec les données géométriques de la configuration étudiée $(L=0,3 \mathrm{~m}$ et $R=10 \mu \mathrm{m})$.

On voit d'après cette figure (Fig. 7) que le débit massique dans le capillaire est d'autant plus faible que la zone de transition est proche de la sortie $(z=0,3 \mathrm{~m})$. La position de la couche limite en fin de parcours a un effet bouchon [13] qui ralentit la progression du fluide vers l'extérieur.

\section{Conclusion}

Dans ce travail de recherche, nous nous sommes intéressés aux problèmes d'étanchéités statiques en situations extrêmes. Plus particulièrement, le cas d'un fluide de Van der Waals qui traverse le point critique sur son chemin. L'approche phénoménologique des équations de Navier Stokes dominées par la viscosité a permis en première approximation de calculer un débit massique. La correction du comportement de l'écoulement a signalé la nécessité d'introduire une couche limite au voisinage de l'état thermodynamique critique en raison de la divergence de certaines propriétés physiques (coefficient de dilatation, conductivité,... .). La modélisation de cette région intérieure à forte dilatation a révélé l'existence d'un couplage thermo-dynamique. Elle a montré également que la convection thermique s'ajoute à la conduction dans cette zone pour assurer le transport de chaleur, contrairement aux régions extérieures amont et aval. Les conditions de raccord nécessaires pour valider la cohérence du modèle établi ont permis de corriger le débit. Les résultats de cette étude ont montré au final que la localisation de la couche limite en fin de parcours d'un chemin de filtration a un effet bouchon qui réduit les fuites du fluide vers l'extérieur.

\section{Références}

[1] J.S. Darrozès, Micro hydrodynamique des étanchéités statiques, Mécanique \& Industries 2 (2001) 299-312

[2] B. Zappoli, D. Bailly, Y. Garrabos, B. Le Neindre, P. Guenoun, D. Beysens, Anomalous heat transport by the piston effect in supercritical fluids under zero gravity, Phys. Rev. A 41 (1990) 2264-2267

[3] A. Onuki, H. Hao, R.A. Ferrel, Fast adiabatic equilibration in a single-component fluid near the liquid-vapor critical point, Phys. Rev. A 41 (1990) 2256-2259

[4] P. Carles, Étude de l'effet piston et des phénomènes thermo-acoustiques dans les fluides supercritiques, rapport de thèse, Institut de mécanique des fluides de Toulouse, 1995

[5] R. Khelalfa, Contribution à l'étude des écoulements de filtration au voisinage du point critique, Thèse, Université Paris VI, 2008

[6] L.P. Kadanoff, J. Swift, Transport coefficient near liquidgas critical point, Phys. Rev. 166 (1968) 89-101

[7] K. Kellner, The critical-point exponent of the thermal conductivity of fluids, J. Phys. D 2 (1969) 1291-1292

[8] A. Jounet, A. Mojtabi, J. Ouazzani, B. Zappoli, Lowfrequency vibrations in a near-critical fluid, Phys. Fluids 12 (2000) 197-204

[9] B. Zappoli, The response of a nearly supercritical pure fluid to a thermal disturbance, Phys. Fluid A 4 (1992) 1040-1048

[10] J.S. Darrozès, C. François, Mécanique des fluides incompressibles, Lecture Notes in Physics, Springer-Verlag, No. 163, 1982

[11] H. Schlichting, Boundary layer theory, 4th edition, McGraw-Hill, 1960

[12] J.S. Darrozès, On the uniformly valid approximate solution of Laplace Equation for an inviscid fluid flow past a three-dimensional thin body, ACR-203 Office of Naval Research, 1973, Vol. 2

[13] V. Feburie et al., A model for choked flow through cracks with inlet subcooling, Int. J. Multiphase Flow 19 (1993) $541-562$ 